\title{
IVERMECTINA E SAÚDE ÚNICA: reposicionamento e a preocupação com o meio ambiente
}

\section{Ivermectin and One Health: repositioning and the concern with the environmental}

Marcelo Beltrão Molento ${ }^{1}$

\author{
*Autor Correspondente: Laboratório de Parasitologia Clínica Veterinária, Departamento de \\ Medicina Veterinária, Universidade Federal do Paraná. R. dos Funcionários, 1540, \\ Curitiba, PR, Brasil. CEP: 80.035-050. \\ E-mail:molento@ufpr.br
}

Como citar: MOLENTO, M. B. Ivermectiva e saúde única: reposicionamento e a preocupação com o meio ambiente. Revista de Educação Continuada em Medicina Veterinária e Zootecnia do CRMV-SP, São Paulo, v. 19, n. 1, 2021, DOl: https://doi.org/10.36440/recmvz.v19i1.38175.

Cite as: MOLENTO, M. B. Ivermectin and One Health: repositioning and the concern with the environmental. Journal of Continuing Education in Veterinary Medicine and Animal Science of CRMV-SP, São Paulo, v. 19, n. 1, 2021, DOl: https://doi.org/10.36440/recmvz.v19i1.38175.

\section{Resumo}

O descobrimento, o sucesso comercial e os benefícios sociais da ivermectina (IVM) sempre foram mundialmente reconhecidos e deu aos seus autores o prêmio Nobel de Medicina/Fisiologia, em 2015. Sua exclusiva ação endectocida e alta segurança permitiram que a IVM fosse imediatamente integrada em vários protocolos sanitários para praticamente todas as espécies animais, incluindo humanos. Entretanto, uso da IVM em larga escala e em alta frequência, acarretou a seleção de parasitos resistentes. Mais recentemente, a IVM vem sendo pesquisada e reposicionada para atuar contra o novo RNA betacorona vírus SARS-CoV-2, principalmente em países da América Latina. Em outra frente, os impactos ambientais também devem ser considerados, pois a eliminação da IVM em grandes quantidades por animais e humanos pode afetar frágeis ecossistemas aquáticos (ex. sedimentos, crustáceos), assim como, comunidades com baixo desenvolvimento social e pouco acesso a redes de água tratada. 0 objetivo da presente revisão foi abordar os potenciais novos usos da IVM, reconhecendo a importância do medicamento e sua atuação na Saúde Única, durante a pandemia COVID-19. Médicos-veterinários podem participar desde momento, orientando o seu uso de maneira cuidadosa para mitigar problemas sanitários em humanos e animais. A avaliação criteriosa pode evitar o uso excessivo da IVM, protegendo organismos não-alvo em todo o ecossistema.

Palavras-chave: Controle de Doenças Parasitárias. Controle Químico. Saúde Humana e Animal.

\section{Abstract}

The discovery, the commercial success, and the social benefits of ivermectin (IVM) were always recognized worldwide and gave the authors the Nobel price of Medicine/Physiology in 2015. Its

1 Docente associado, Universidade Federal do Paraná, Departamento de Medicina Veterinária, Laboratório de Parasitologia Clínica Veterinária, Curitiba, PR, Brasil 
exclusive endectocide activity and great safety allowed IVM to be immediately integrated in various health protocols to practically all animal species, including humans. Even tough, the large-scale and the high frequency of use of IVM has caused the selection of resistant parasites. More recently, IVM is being searched and repositioned to act against the new SARS-CoV-2 virus, mainly in Latin American countries. In another front, environmental protection must also be considered, as the large IVM elimination by humans and animals may affect fragile marine ecosystems (ex. sediments, crustaceans) as well as, unassisted communities with low social development with low access to safe water. The objective of the present review was to cover the potential new uses of IVM recognizing the importance of the compound to One Health, during the COVID-19 pandemic. Veterinarians can participate in this moment by advising its correct use to mitigate health problems in humans and animals. A careful evaluation may avoid the excessive use of IVM protecting non-target organisms on the entire ecosystem.

Keywords: Parasitic Diseases. Welfare. Chemical Control. Health Systems. Planetary Health.

\section{Introdução}

A ivermectina (IVM, C48H74014) é um medicamento derivado do fungo Streptomyces avermitilis, com alto peso molecular $(875.1 \mathrm{~g} / \mathrm{mol})$ e que faz parte da família das avermectinas. 0 fármaco teve origem a partir de processos de fermentação e foi estruturado com a combinação de dois componentes (B1a/80\% e B1b/20\%) (Figura 1). A IVM tem sido amplamente utilizada na sua administração oral ou injetável para o controle de doenças, tanto na agricultura e nos animais, como também em humanos, desde os anos 70 (CARTER; NIETSCHE; BORDERS, 1987; FISHER; MROZIK, 1992). A IVM faz parte da única família de fármacos com ação endectocida, que são as avermectinas e as milbemicinas, englobando os importantes compostos semissintéticos; moxidectina (S. hydroscopicus aureolacrimosus), abamectina, doramectina, eprinomectina e selamectina, principalmente. Esta família de fármacos apresentou uma eficácia acima de $98 \%$ contra importante parasitos de animais e humanos, como Haemonchus contortus (verme da papeira), Dirofilaria immitis (verme do coração), a sarna Sarcoptes scabiei e ainda uma ação significativa contra a mosca Haematobia irritans (moscados-chifres) e o carrapato do boi, Rhipicephalus microplus (BOOTH; MCDONALD, 1988).

Em parasitos nematodas, o mecanismo de ação da IVM ocorre quando a molécula se liga e ativa a sub-unidade alfa do canal de cloro potencializado pelo glutamato (GluCl). Este local de ação é expresso em todas as células nervosas e na musculatura da faringe destes e de outros organismos invertebrados. A IVM atua também em canais de cloro mediados pelo ácido gama-aminobutírico (ARENA et al., 1995). Nestes sítios, a IVM causa o efeito de hiperpolarização e paralisia flácida, com a morte e eliminação dos vermes (DENT; DAVIS; AVERY, 1997). Comercialmente, a IVM foi lançada no início dos anos 80, causando um impacto positivo, jamais visto na área da terapêutica veterinária mundial. Desde aquele tempo, o produto foi alçado ao patamar de químicos como a morfina, aspirina e penicilina, lançadas em 1827, 1899 e 1942, respectivamente, devido às suas características de segurança, seu amplo espectro contra endo e ectoparasitos e seu exclusivo efeito de longa ação (MOLENTO, 2020) em formulações específicas para animais. Muito embora exista grande margem de segurança, a molécula da IVM se liga na musculatura, fígado e tecido adiposo do hospedeiro, sendo lentamente eliminada em $96 \%$ por via fecal (BOOTH; MCDONALD, 1988). Todas estas vantagens terapêuticas trouxeram mais segurança para as iniciativas One World - One Health, Planetary Health e Global Health. 
Figura 1 - Estrutura molecular da ivermectina. As principais diferenças entre moléculas da mesma família, são observadas na posição do carbono 13 e 25 (círculos vermelhos)

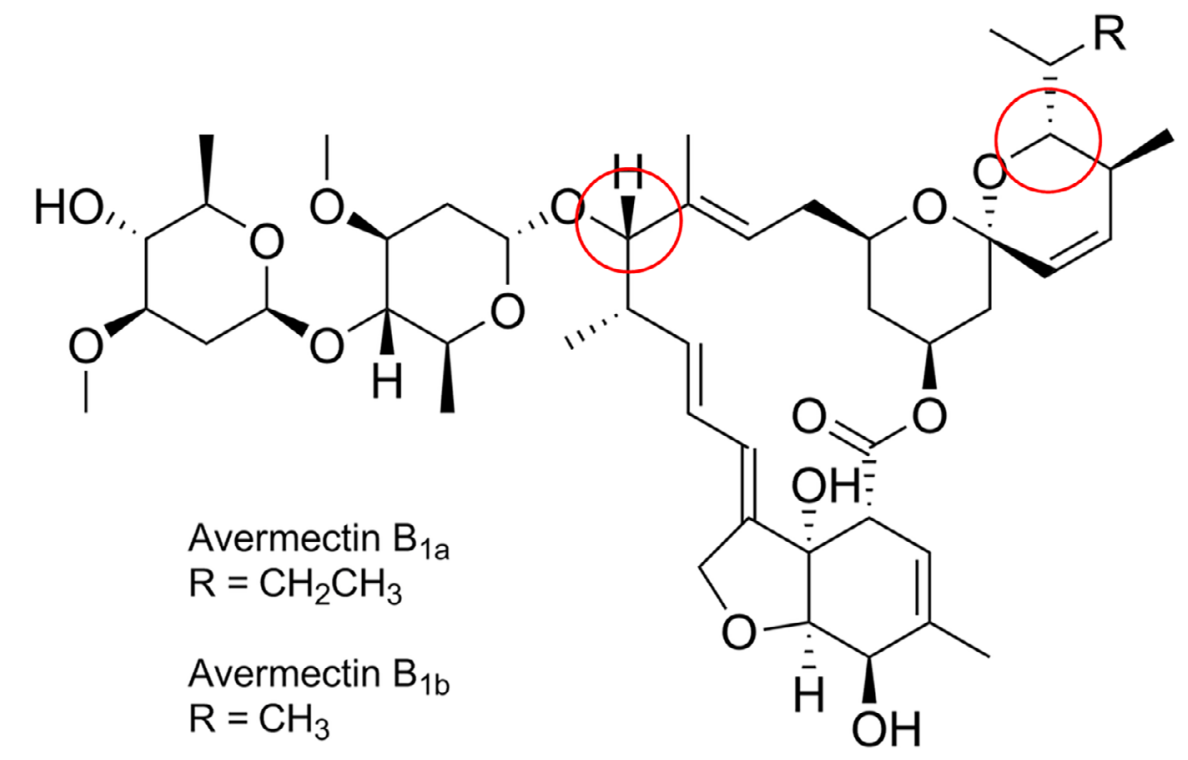

Fonte: Carter, Nietsche e Borders (1987).

O impacto causado por doenças parasitárias, como Fasciola hepatica e outras, pode ser medido nos animais aplicando-se protocolos de bem-estar e realizando exames clínicos específicos e laboratoriais, com grande margem de acerto. 0 impacto econômico das parasitoses também é sentido nas comunidades que dependem da atividade pecuária, podendo alcançar cifras de milhões de dólares (MOLENTO et al., 2020). Em humanos, as infecções parasitárias (ascaridíase, ancilostomíase, cisticercose, giardíase) atingem crianças e jovens, afetando diretamente comunidades vulneráveis, com pouco acesso ao saneamento básico, como redes de esgoto e água tratada (GARCIA, 2017). A mensuração do impacto destas e de outras doenças de base, também pode ser medida pelo valor de DALY que é uma medida de "ano de vida ajustado por incapacidade". Este formato de avaliação permite uma melhor definição de políticas públicas, comparando populações e condições de saúde ao longo do tempo, incluindo aí, a avaliação de "anos perdidos por sequelas e mortalidades" (YLL). Torgerson et al. (2015) realizaram uma revisão sistemática sobre doenças parasitárias, incluindo 10 helmintoses e toxoplasmose, transmitidas por alimentos em âmbito regional e global. 0 cálculo dos autores revelou que estas doenças causaram 6,6 milhões de DALYs, sendo a cisticercose, a doença de maior impacto em humanos, com 2,9 milhões.

O terceiro fator que engloba a iniciativa de Saúde Única é seguramente o mais complexo e importante, referindo-se ao componente ambiental do uso da IVM. A presença deste fármaco no meio ambiente, seu potencial tóxico e consequências em médio e longo prazo estão sendo estudadas desde os anos 80. Entretanto, as concentrações de IVM no ambiente têm sido pouco avaliadas, principalmente nas regiões produtoras de alimentos, como na África e na América Latina, assim como em países que usam a IVM de modo preventivo em animais de companhia (MANCINI et al., 2020). Como dito anteriormente, o fármaco ativo é eliminado praticamente integro nas fezes e por isto não se observa claramente os danos causados em curto prazo, porém, como sua liberação é lenta e cumulativa por mais de 200 dias, os danos ambientais são definitivamente muito preocupantes (MESA et al., 2020).

0 presente artigo tem o objetivo de atualizar dados em relação a IVM, abordando seu uso como um produto singular para aplicação em Saúde Única, enriquecendo e valorizando o papel do médicoveterinário em momentos cruciais para toda a sociedade. Outro ponto importante é abordar o potencial de reposicionamento da IVM no contexto da pandemia Covid-19, causada pelo novo coronavírus. 


\section{Ivermectina: um medicamento para a humanidade}

Os Drs. Satoshi Ômura e William Campbell receberam o prêmio Nobel de Medicina/Fisiologia em 2015, pelo uso da IVM em programas continentais de combate a doenças parasitárias desde a década de 1980. Após esta iniciativa, a IVM realmente ganhou um status especial e grande reputação, quando se descobriu seu potencial para reduzir e até mesmo erradicar, doenças parasitárias em humanos e animais. Existem alguns exemplos práticos, como o Dictyocaulus viviparus que, praticamente, desapareceu em regiões com criações de ovinos no estado de Santa Catarina (RAMOS et al., 2004) e dos grandes estrôngilos em equinos (PEREGRINE et al., 2014), em várias partes do mundo. Mesmo antes de ter seu uso registrado para humanos, sua ação endectocida foi muito bem recebida pela comunidade científica veterinária.

Em uma das obras mais importantes no campo da medicina preventiva, o Instituto Merck tem realizado doações anuais de IVM para combater a cegueira dos rios causada por Onchocerca volvulus, melhorando a condição de vida de milhões de pessoas na África e em outros lugares do mundo, incluindo o Brasil - o mesmo acontecendo com a filariose linfática (KUESEL, 2016). Inclusive, o senador americano Ted Kennedy afirmou, em 1987, que "o presente da empresa Merck para a Organização Mundial de Saúde é mais do que um avanço da Medicina - é um verdadeiro triunfo ao espírito da humanidade." Há uma estimativa de que mais de três bilhões de doses já tenham sido distribuídas nestes programas. Mais recentemente, pesquisadores publicaram novos avanços para o uso de IVM em um encontro da Associação Americana de Medicina Tropical, criando a "Rede de Eliminação da Malária com Ivermectina". Esta iniciativa representa uma série de pesquisas com o uso de IVM para definir sua ação contra o(s) mosquito(s) vetor(es) da doença (CHACCOUR et al., 2015). 0 maior desafio para este caso está no ajuste das doses de IVM, visto que são necessárias concentrações elevadas do fármaco na circulação sanguínea de humanos, mantendo os altos níveis de segurança, e que, ao mesmo tempo, atinjam concentrações letais que afetem Aedes aegypti (HADLETT et al., 2021). Novamente, esta iniciativa e tantas outras ações com o uso do medicamento, conferem a IVM um patamar bastante diferenciado no cenário terapêutico mundial.

\section{Resistência: uma corrida intensa, longa e vitoriosa -} até certo ponto!

O controle das infecções parasitárias é extremamente necessário para humanos e animais, pois resulta em uma melhor qualidade de vida para todos. Entretanto, o uso exclusivo de compostos antiparasitários (ex. benzimidazóis, imidazotiazóis e lactonas macrocíclicas), gerou muita dependência e pouca diversificação de estratégias sanitárias nos programas de prevenção (MOLENTO, 2009). Uma vez lançada a IVM, foi observado uma corrida para seu uso e difusão em larguíssima escala. Profissionais adotaram uma grande frequência de uso do medicamento em todos os programas de saúde animal, incluindo ruminantes, suínos, equinos e animais de zoológico. Durante mais de duas décadas, o uso intensivo de IVM representou aproximadamente $80 \%$ do mercado farmacêutico para animais de produção, onde todos os envolvidos estavam confiantes, conseguindo eficácias excepcionais. Vale lembrar, que nenhum antiparasitário é eficaz contra $100 \%$ dos estádios de desenvolvimento dos parasitos, em $100 \%$ das aplicações e em $100 \%$ dos animais. De fato, assim como ocorreu em situações semelhantes desde os anos 60 com os benzimidazóis, a IVM apresentou uma visível queda de eficácia após alguns anos de uso (COLES et al., 2003).

Tratamentos em curto espaço de tempo, o uso de altas concentrações dos produtos e o tratamento de todos os animais do rebanho, são as práticas mais recomendadas na pecuária e também são considerados os maiores fatores de risco para a seleção de parasitos resistentes em rebanhos (PRICHARD, 2001). Contudo, estas estratégias de tratamento consideradas supressivas, 
com vistas à erradicação de parasitos tiveram de ser repensadas, devido a clara evidência da baixa eficácia dos produtos, incluindo a IVM (TORRES-ACOSTA; MOLENTO; DE GIVES, 2012). Em humanos, o tratamento químico massivo nas populações afetadas também impactou na seleção de parasitos resistentes, como foi o caso de Onchocerca volvulus (OSEI-ATWENEBOANA et al., 2011). Os primeiros relatos de resistência deste parasito, incentivaram os agentes de saúde a implementar ações voltadas para a determinação de focos ativos da doença e o controle de vetores. No Brasil, a população em risco e de transmissão ativa da oncocercose é de aproximadamente 15 mil pessoas e se concentra exclusivamente nas tribos lanomâmis, na região norte da Amazônia, divisa com a Venezuela. Este é o único foco ativo da doença nas Américas e os indígenas necessitam de até quatro tratamentos com IVM ao ano, devido ao caráter hiperendêmico da doença em mais de 100 comunidades. 0 mais difícil neste caso é o habito migratório das tribos e seu difícil tratamento, sendo necessária a participação de agentes médicos indígenas (SAUERBREY et al., 2018).

Atualmente, a resistência contra os fármacos é um dos problemas mais sérios para a manutenção da saúde animal e humana, devido, inclusive, ao compartilhamento do mesmo medicamento (ex. IVM/ helmintoses e escabioses e triclabendazole/F. hepatica) entre diferentes hospedeiros (COBO, 2016; MAS-COMA et al., 2020). A queda na eficácia da IVM foi determinada em vários países na década de 1990 e logo foram determinados os primeiros mecanismos moleculares da resistência, como o sítio de ligação no GluCl (DENT; DAVIS; AVERY, 1997), permitindo o diagnóstico precoce da resistência e a possível manutenção de sua eficácia. Todavia, mesmo existindo a possiblidade de monitorar a eficácia da IVM e demais medicamentos, esta prática é pouco utilizada e, normalmente, a resistência é avaliada após a observação empírica do pouco efeito da medicação, com o comprometimento da saúde dos animais.

Alguns testes de eficácia de anti-parasitários podem ser realizados in vitro, avaliando a eclodibilidade de ovos e a motilidade e o desenvolvimento de larvas de parasitos nematodas (DEMELER et al., 2010). Os testes descritos acima já são realizados em vários laboratórios especializados no Brasil. 0 teste controlado permite a comparação de um grupo controle de animais infectados sem tratamento com um grupo infectado e tratado, nos períodos de pré e pós-tratamento. No caso dos testes in vivo, se utiliza a técnica de contagem de ovos de parasitos por grama de fezes (OPG) para determinar o percentual de eficácia de medicamentos. Novos métodos empregando a biologia molecular (ex. PCR, qPCR) e a nanotecnologia podem trazer maior agilidade ao diagnóstico e novas formas de terapias (MOLENTO; ARENAL, 2020), permitindo abordagens inéditas na busca por mecanismos de ação e interações sinérgicas, dos medicamentos antiparasitários com agentes potencializadores (BORTOLUZZI et al., 2021).

Estratégias de controle parasitário com menor uso de produtos químicos buscando a redução de IVM em até $90 \%$, também são importantes possibilidades para retardar a seleção de parasitos resistentes. Dentre elas, existe a identificação clínica de animais resilientes, fortalecendo a avaliação seletiva (MOLENTO et al., 2013) e o uso individualizado de medicamentos, assim como está ocorrendo para o controle de uso dos antibióticos (DOMAN, 2019). Todos estes cuidados estão em sintonia com a iniciativa de Saúde Única, inclusive para a proteção de ecossistemas. Mais estudos são necessários para validar tais estratégias, contrapondo o possível aumento no custo de mão de obra e manejo nas condições de rebanhos no Brasil. Muito embora, tais fatores causariam menor impacto, quando são comparados ao desenvolvimento de parasitos resistentes e os danos ao meio ambiente com o uso excessivo de químicos.

\section{Ivermectina, um fármaco de amplo espectro e multialvo}

$\mathrm{Na}$ farmacologia, se considera que uma das melhores qualidades de um medicamento é saber que ele atua de forma específica e em um único sítio de ação. Essa característica evita efeitos 
adversos desconhecidos, deixando de interagir em outros sítios e em locais e organismos não-alvos. Entretanto, após a anotação do genoma humano, muito se avançou na área farmacológica, com a busca de novos alvos em várias doenças. Assim, terapias mais modernas preferem a atuação de medicamentos contra doenças multifatoriais, envolvendo múltiplos alvos (MAKHOBA et al., 2020). A característica multialvo da IVM tem sido explorada desde a sua descoberta, quando ainda não se valorizava muito esta característica. Tais descobertas causaram grande entusiasmo devido ao sucesso em experimentos in vitro e in vivo contra o vírus da AIDS e alguns tipos de câncer, bactérias e até na doença de Alzheimer (ZBARSKY; THOMAS; GREENFIELD, 2004). Fármacos multialvo como a IVM, apresentam uma abordagem moderna e eficaz e a Agência Regulatória Americana (Food and Drug Administration, FDA), pondera sobre pedidos de registro de tais produtos, reconhecendo os benefícios e os riscos para pacientes acometidos por doenças multifatoriais (LU et al., 2012).

Em uma busca recente no SCOPUS, o maior banco de dados de artigos científicos, foi constatada a existência de mais de 14 mil trabalhos com IVM, dos quais muitos, provenientes de pedidos de patentes (SCOPUS, 2021) (Figura 2). Os países que mais registraram estes pedidos foram os EUA, Japão e países Europeus, com 64, 24 e 7\%, respectivamente. Foram pesquisadas várias doenças, como vírus e bactérias, incluindo câncer (1330/3776) e depressão (213/1267). Foi relevante observar que a IVM segue despertando grande interesse, não somente por sua ação como antiparasitário com mais de cinco mil citações. Os estudos em humanos são imensamente (>500\%) mais frequentes do que estudos em animais (Figura 2).

Também foi observado que os registros de patente foram mais numerosos do que os artigos científicos. Isto ocorre, principalmente, porque muitas pesquisas estão sendo desenvolvidas em laboratórios de indústrias farmacêuticas, com a circulação restrita de dados. Desta forma, além de preservar a originalidade da descoberta, a corrida segue na busca por novas ações para a molécula multialvo. Outro ponto importante, é que não foi observada uma correlação entre os dados de registro de propriedade intelectual e os produtos comerciais (ex. ausência de IVM como antiviral). Isto se deve ao alto custo para finalizar o processo de registro, ao longo tempo, para a comprovação da fase 4 e a possível resposta do mercado, não sinalizando o retorno do investimento, mesmo considerando problemas de saúde pública (ex. parasitos, vírus e bactérias).

Figura 2 - Área de pesquisa e número de trabalhos que incluíram ivermectina no título e no resumo (colunas pretas) e as ocorrências de registro de propriedade intelectual (Patentes) (colunas cinzas)

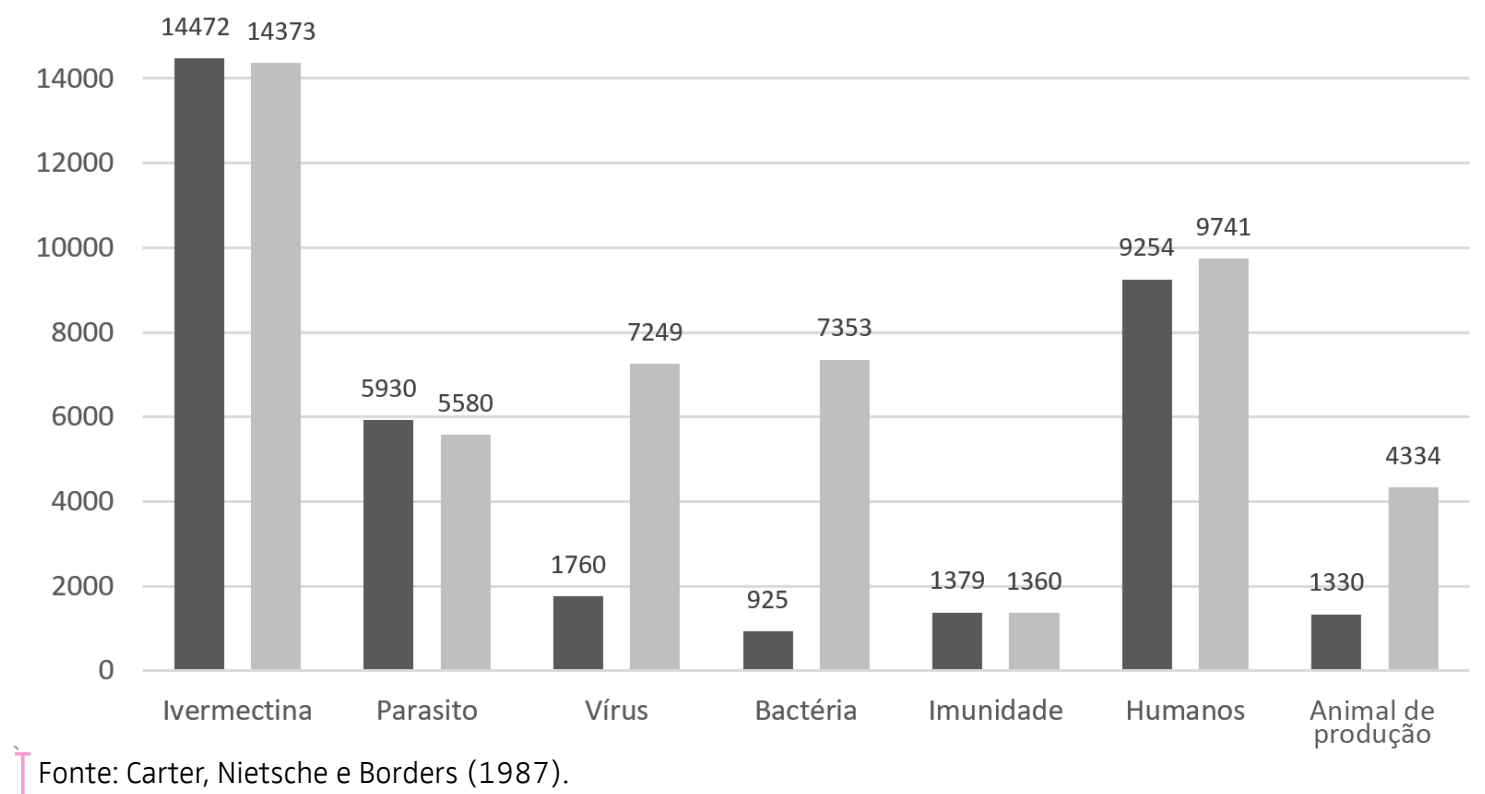


No caso específico da IVM, existe um grande interesse por novas descrições de sua eficácia e novos alvos, devido a recente pandemia causada pela síndrome respiratória do novo RNA beta-corona vírus, SARS-CoV-2 e a publicação do seu efeito antiviral in vitro inibindo a replicação do vírus obtido com altas doses de IVM em um estudo na Austrália (CALY et al., 2020). Existem 18 estudos públicos e privados que estão sendo realizados em ambulatórios em várias partes do mundo e podem ser acompanhados periodicamente (UNITED STATES, 2020). A iniciativa do NIH é muito ampla, mantendo dados atualizados da Covid-19, incluindo os estudos clínicos finalizados com IVM e os que ainda estão em fase de recrutamento ambulatorial em escala mundial.

\section{Ivermectina e a pandemia Covid-19: potencial para reposicionamento}

Em dezembro de 2019, o mundo ficou sabendo do vírus SARS-CoV-2, que abalou a estrutura sanitária de vários países. 0 impacto da doença fez com que governos adotassem o bloqueio de fronteiras, o isolamento social e o fechamento de estabelecimentos comerciais e de atividades públicas por vários meses. Laboratórios no mundo todo começaram a pesquisar medicamentos para enfrentar a nova guerra sanitária (WEHBE et al., 2021). Um dos primeiros relatos científicos veio do Royal Melbourne Hospital, na Austrália, no qual os autores sugeriram que a IVM poderia atuar, competindo com o sítio de ação da Importina-Beta1, que é uma proteína de transporte, eliminando o vírus em até $48 \mathrm{~h}$ (CALY et al., 2020). Os resultados, mesmo sendo realizados em células, repercutiram de forma rápida, muito devido à possibilidade do uso em humanos, o que tornou a IVM em um dos primeiros medicamentos usados em larga escala internacionalmente, mesmo sem a comprovação clínica. Os próprios autores alertaram sobre as limitações dos testes in vitro, porém mesmo com estes alertas, a IVM tem sido utilizada em ambulatórios e em pesquisas cientificas contra a Covid-19. Na atualidade estudos clínicos estão sendo conduzidos na Argentina, Egito, Iraque, México, Paquistão, Índia, Estados Unidos, Austrália e no Brasil, (AGÊNCIA NACIONAL DE VIGILÂNCIA SANITÁRIA, 2021).

É fundamental que a população tenha dados concretos sobre a ação da IVM e profissionais da Medicina Veterinária devem participar ativamente neste processo, auxiliando com informações técnicas e ações terapêuticas do produto para toda a comunidade. A absorção da IVM em humanos é claramente diferente dos animais, fato este ligado à formulação e todo o processo de preparação do composto, tendo como consequência diferenças no mecanismo de ação, na farmacocinética e na eficácia clínica da IVM neste hospedeiro (IKEDA, 2003). A absorção em humanos atinge a concentração máxima no plasma entre 20 a $50 \mathrm{ng} / \mathrm{ml}$, após a administração de 6 a $12 \mathrm{mg} / \mathrm{kg}$, respectivamente. 0 tempo para alcançar a concentração máxima é de aproximadamente 4h, com uma meia-vida de eliminação de 12 a 24h, sendo majoritariamente excretada nas fezes, com raríssimos casos de intoxicação ou efeitos adversos (CANGA et al., 2008). Entretanto, foram descritos sinais neurológicos com o uso da IVM (ex. confusão mental, tremores, desmaio, vômito e diarreia) e que podem persistir por até uma semana. Altas doses administradas em camundongo (DL50 $>27 \mathrm{mg} / \mathrm{kg}$ ) causaram efeitos adversos na pele e olho, fertilidade e reprodução (teratogenia), e toxicidade hepatocelular (OTTENSEN; CAMPBELL, 1994).

0 fato da IVM se ligar em diferentes locais de diferentes proteínas, incentivou a continuidade da busca por um tratamento antiviral eficiente (SÍMSEK; YAVUZ; ÜNAL, 2020). Assim, hospitais ligados a centros de pesquisa e universidades no mundo todo, iniciaram pesquisas com o objetivo de controlar a Covid-19, utilizando medicamentos já aprovados, como antivirais ou não, por agências sanitárias governamentais, como a FDA e a Anvisa, para usá-los em protocolos contra a nova infecção. Entretanto, os dados de eficácia da IVM ainda não foram comprovados com ação antiviral e a atitude de uso massivo, com o consumo de 43 milhões de caixas do produto, no ano de 2020, foi principalmente devido à fragilidade dos sistemas de saúde em países do mundo todo e o medo de estar enfrentando algo extremamente letal (MOLENTO, 2020). 
A estratégia de reposicionamento (repositioning ou repurposing) de produtos é frequentemente utilizada com medicamentos multialvo e o exemplo da IVM, já licenciada no mundo todo e eficaz contra uma série de doenças, inclusive com patentes requeridas como antiviral, é um dos exemplos mais promissores (LAING; GILLAN; DEVANEY, 2017). As agências reguladoras exigem que exista alguma evidência clínica (eficácia, segurança e qualidade) do produto, para que possa ser realizado o pedido formal para extensão de uso deste fármaco. Para que exista o registro, é necessário que se cumpram todos os requisitos listados anteriormente, de acordo com os parâmetros estabelecidos pela Federação Europeia de Indústrias Farmacêuticas (WEHBE et al., 2021). Caso contrário, o uso do medicamento será chamado de 'off-label' e não terá licença para sua nova proposta de emprego. Nesta situação, a denominação "off-labels" se refere a medicamentos aprovados e eficazes contra alguma doença, porém ainda sem a revisão e autorização dos dados (SCHMITH; ZHOU; LOHMER, 2020).

Muito embora alguns estudos tenham demonstrado o efeito da IVM, juntamente com o zinco e a vitamina C, na redução da carga viral em pacientes assintomáticos, os vírus de RNA, como o SARSCoV-2, são capazes de apresentar mutações rapidamente (SANTOS et al., 2020) e o uso isolado da IVM não pode ser descartado como um fator central de seleção de estirpes altamente patogênicas e resistentes (WEHBE et al., 2021).

\section{Ivermectina no meio ambiente: um assunto bastante delicado}

Como relatado, a IVM é eliminada na sua maioria (>96\%) inalterada nas fezes e em grandes quantidades. Utilizando dados de questionários sanitários sobre a frequência de tratamentos no Brasil, a eliminação de IVM no ambiente pode ser de aproximadamente 15 toneladas por ano (MOLENTO, 2009). A presença de IVM no esterco pode ser nociva para muitas espécies de besouros coprófagos, tanto na abundância, quanto na diversidade destes insetos. Galbiati et al. (1995), demonstraram que a IVM e a doramectina causaram maior taxa de mortalidade de besouros Dichotomius anaglypticus artificialmente inoculados nas fezes, após uso injetável dos produtos em bovinos, comparados ao controle. Após a eliminação nas fezes, a IVM chega rapidamente ao solo, sedimentos e na raiz de plantas, efeito este que pode persistir por até 200 dias (FISHER; MROZIK, 1992). A partir daí, a IVM chega também em cursos de água e lagoas, com a chuva, ameaçando seriamente demais organismos não-alvo e ecossistemas (MESA et al., 2020) (Figura 3).

A concentração da IVM eliminada no bolo fecal pode chegar a $0,1 \%$ do medicamento $(0,2 \mathrm{ppb}-$ partes por bilhão), após o tratamento de um bovino adulto, sabendo que a concentração tóxica da IVM em organismos aquáticos é de $3 \mathrm{ppb}$ em crustáceos, de 5 ppb em peixes e de 200 ppb para fungos e bactérias (WANG et al., 2019). Desta forma, seria necessário tratar 1.000 animais para atingir os níveis de toxicidade "local" para todas estas espécies, ou tratar 200 animais todo o mês, fato que ocorre regularmente em criações de pequenos ruminantes e equinos. Na mesma conta, vários insetos e outros invertebrados podem ser afetados pelo resíduo de IVM após o tratamento dos animais. Além destes, as espécies não-alvo necessitam ser melhor estudadas em laboratório (ex. Daphnia magna), inclusive para monitorar e avaliar a amplitude da disseminação da molécula e sobre seu uso e impacto inclusive para a aquicultura comercial. A fauna afetada por altas concentrações de IVM pode ser fundamental aos ciclos de fertilidade do solo e para a cadeia alimentar de espécies não-alvo mais sensíveis, como garças e falcões (MESA et al., 2020).

Outro dado importante é que os danos causados pela bioacumulação da IVM já foram mensurados e deve-se a sua longa persistência, baixa degradação e ampla distribuição em organismos presentes no bolo fecal (FISHER; MROZIK, 1992). A partir destas evidências, ficou claramente comprovada, a necessidade de se revisar a estratégia dos tratamentos preventivos dos rebanhos, incluindo os programas estratégicos com datas fixas (PEREGRINE et al., 2014; TORRES-ACOSTA; MOLENTO; DE GIVES, 2012). 0 objetivo de 
estratégias com base epidemiológica e clínica é de reduzir o impacto causado nos ecossistemas, muitos deles reconhecidamente frágeis, como é o caso de regiões do Pantanal, Cerrado, Mata Atlântica e na vasta região Amazônica, ocupadas com a grande presença de bovinos. Outra forma sutil e danosa ao meio ambiente é a chamada bioampliação dos produtos a partir da bioacumulação em insetos ou outros organismos (VERDÚ et al., 2020). Neste caso, o acúmulo de IVM em tecidos de besouros ou minhocas, por exemplo, permite que o produto fique protegido e persista ativamente por até duas vezes mais tempo, amplificando seu raio de interação, podendo chegar a toda a cadeia alimentar local (Figura 3).

A IVM é rapidamente distribuída na matriz água e em um dia após a sua administração nos animais, pode ocorrer o seu acúmulo no lodo de rios e represas, resultando em resíduos de longa duração (>400 dias). Sabendo que no Brasil o desafio parasitário é constante e que o regime de tratamento em animais de produção é realizado em quase sua totalidade com o uso de químicos e em intervalos curtos (< 90 dias), a IVM que alcança o ambiente irá apresentar grande acúmulo, antes mesmo da sua degradação. No Paraná, Fernandes et al. (2017), determinaram que ovinos que não receberam moxidectina, uma macrolactona semelhante a IVM, apresentaram traços deste químico no fígado e na gordura, após pastejarem na mesma área de animais tratados com a dose recomendada pelo fabricante. Os autores apresentaram a hipótese de que esta contaminação ambiental possa ser estendida para outros animais que dividem a área dos ovinos, incluindo herbívoros e roedores selvagens. Devido as semelhanças estruturais, esses danos podem ser semelhantes para a IVM. Esta suspeita necessita ser comprovada com a realização de novos estudos.

Para evitar tais abusos, a Comissão Europeia estabeleceu normas de regulamentação, com níveis de toxicidade para vários medicamentos, após a avaliação do impacto de produtos, como a IVM e seu uso massivo veterinário (MANCINI et al., 2020) e que deverão ser atingidos até 2030 (EUROPEAN MEDICINES AGENCY, 2008). Para definir tais padrões, Mancini et al. (2020) realizaram modelos matemáticos para determinar cenários de risco, incluindo o impacto em humanos, com a eliminação de fármacos nos efluentes e o consumo de água não tratada. No Brasil, é imperativo que se discutam estas possibilidades, visando um melhor monitoramento destes resíduos, incluindo acaricidas, que apresentam alto risco tóxico ambiental e têm uso frequente. Somente assim, será possível o estabelecimento de medidas de uso consciente de frequência e concentração, reduzindo os efeitos adversos, previstos nos protocolos de farmacovigilância, para se trabalhar em consonância com as iniciativas de Saúde Única, EcoHealth e Planetary Health.

Figura 3 - Percurso em dias da ivermectina no ambiente, incluindo os efeitos de bioacumulação e bioampliação

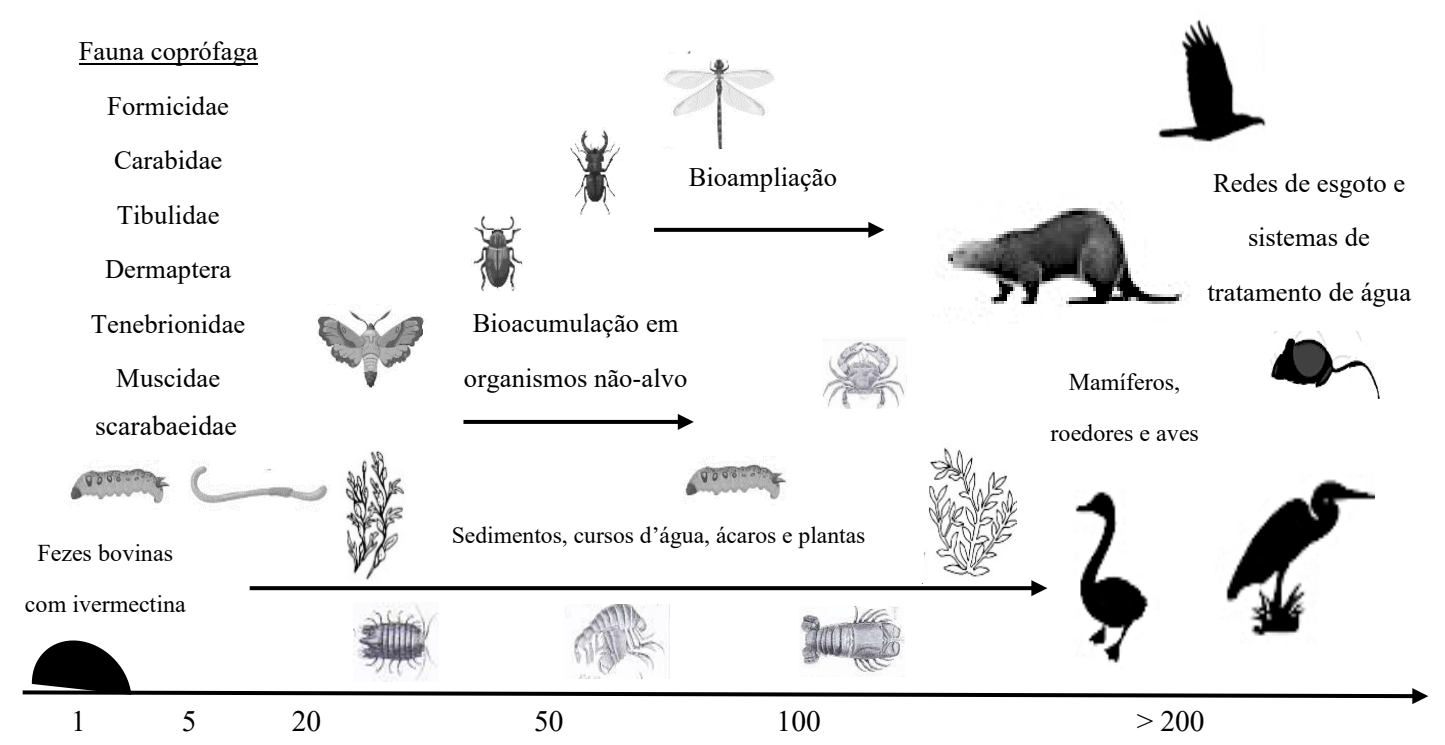

TFonte: Adaptado de Fisher e Mrozik (1992), Mesa et al. (2020) e Verdú et al. (2020). 
Profissionais veterinários devem ter a preocupação de recomendar o uso de IVM e similares somente após uma criteriosa análise clínica, acompanhada por exames laboratoriais complementares com aplicações seletivas. Esta atitude poderá reduzir significativamente o número de tratamentos antiparasitários, permitindo ainda avaliar a capacidade resiliente de alguns animais no rebanho, baseada no limite transitório de infecção (Transient treshold abundance - TTA) (SCHAFASCHEK et al., 2021). Estes esforços no campo, juntamente com a legislação existente nacional, poderão auxiliar na proteção e manutenção de baixos níveis desses produtos no ambiente, evitando que se chegue ao limite de banir a sua comercialização.

\section{Conclusão: conscientização, vida longa e muitas}

\section{pesquisas}

A IVM é um medicamento multialvo fundamental para a manutenção da saúde de humanos e animais, mesmo tendo mais de 40 anos de uso contínuo. Novos mecanismos de ação deverão ser revelados para este fármaco, permitindo a cura e o alívio de muitas doenças. 0 combate à Covid-19 ainda está muito difícil, porém o reposicionamento da IVM poderá permitir um alívio ao sistema de saúde do Brasil e de outros países, embora ainda existam grandes gargalos tecnológicos e estruturais para serem transpostos. Ainda sobre este tema, o uso preventivo da IVM em humanos deverá ser regulamentado por agências governamentais, após o cumprimento da legislação pertinente, exigindo um rígido acompanhamento. A regulação da utilização da IVM deve ter também sólido apoio científico e ampla comprovação ambulatorial. Campanhas de conscientização para uso de medidas preventivas e a vacinação em massa também são essenciais para a manutenção da saúde da população.

Médicos-veterinários têm o dever de desenvolver, difundir e aprender novas estratégias de uso dos medicamentos, permitindo que a Medicina Veterinária Preventiva olhe para os animais de maneira holística, auxiliando na implantação de estratégias de Saúde Única. A Medicina Veterinária é independente para atuar em áreas fundamentais da saúde animal e humana, fortalecendo o compromisso com o bemestar das comunidades, tendo como objetivos centrais o auxílio no controle de doenças, a redução do sofrimento animal e a utilização de práticas sustentáveis ao meio ambiente.8

\section{Referências}

AGÊNCIA NACIONAL DE VIGILÂNCIA SANITÁRIA. Lista de ensaios clínicos com medicamentos para prevenção ou tratamento da COVID-19 autorizados pela Anvisa. 2021. Disponível em: https:// www.gov.br/anvisa/pt-br/assuntos/medicamentos/pesquisaclinica/arquivos/ensaios-clinicos-covid. pdf. Acesso em: 1 jun. 2021.

ARENA, J. P. et al. The mechanism of action of avermectins in Caenorhabditis elegans: correlation between activation of glutamate-sensitive chloride current, membrane biding and biological activity.

Journal of Parasitology, [s.I.], v. 81, p. 286-294, 1995.

BOOTH, N. H.; MCDONALD, L. E. Veterinary pharmacology and therapeutics. 6th. ed. [s.I.]: lowa State University Press, 1988. p. 916-917.

BORTOLUZZI, B. B. et al. Mentha villosa Hubs., M. x piperita and their bioactives against gastrointestinal nematodes of ruminants and the potential as drug enhancers. Veterinary Parasitology, [s.l.], v. 289, 109317, 2021. DOI: https://doi.org/10.1016/j.vetpar.2020.109317.

CALY, L. et al. The FDA-approved drug ivermectin inhibits the replication of SARS-CoV-2 in vitro.

Antiviral Research, [s.I.], 2020. DOI: https://doi.org/10.1016/j.antiviral.2020.104787. 
CANGA, A. G. et al. The pharmacokinetics and interactions of ivermectin in humans: a mini-review. American Association of Pharmaceutical Scientists, [s.l.], 2008. DOl: https://doi.org/10.1208/ s12248-007-9000-9.

CARTER, G. T.; NIETSCHE, J. A.; BORDERS, D. B. Structure determination of LL-F28249 alfa, beta, lambda potent antiparasitic macrolides from Streptomices cyanogriceus spp. noncyanogenus. Journal of the Chemical Society, [s.I.], v. 3, p. 402-404, 1987.

CHACCOUR, C. J. et al. Establishment of the ivermectin research for malaria elimination network: updating the research agenda. Malaria Journal, [s.I.], v. 14, p. 243-251, 2015. DOI: https://doi. org/10.1186/s12936-015-0691-6.

COBO, F. Determinants of parasite drug resistance in human lymphatic filariasis. Revista Española de Quimioterapia, [s.I.], v. 29, p. 288-295, 2016.

COLES, G. C. et al. Anthelmintic resistance and the use of anthelmintics in horses. Veterinary Record, [s.I.], v. 162, p. 636, 2003.

CORSELLO, S. M. et al. The drug repurposing hub: a next-generation drug library and information resource. Nature Medicine, [s.I.], v. 23, p. 405-408, 2017. DOI: https://doi.org/10.1038/nm.4306.

DEMELER, J. et al. Standardization of the larval migration inhibition test for the detection of resistance to ivermectin in gastro intestinal nematodes of ruminantes. Veterinary Parasitology, [s.l.], v. 174, p. 58-64, 2010. DOI: https://doi.org/10.1016/j.vetpar.2010.08.020.

DENT, J. A.; DAVIS, M. W.; AVERY, L. Avr-15 encodes a chloride channel subunit that mediates inhibitory glutamatergic neurotransmission and ivermectin sensitivity in Caenorhabditis elegans. EMBO, [s.l.], v. 16, p. 5867-5879, 1997.

DOMAN, E. McDonald's new CEO: how Chris Kempczinski can impact the future of chicken welfare. The Human League, [s.I.], 2019. Disponível em: https://thehumaneleague.org/article/mcdonalds-chriskempczinski. Acesso em: 11 maio 2021.

EUROPEAN MEDICINES AGENCY. Revised guideline on environmental impact assessment for veterinary medicinal products in support of the VICH guidelines GL6 and GL38. London, UK: Committee for Medicinal Products for Veterinary Use (CVMP), 2008.

FERNANDES, M. A. M. et al. Moxidectin residues in tissues of lambs submitted to three endoparasite control programs. Research in Veterinary Science, [s.I.], v. 114, p. 406-411, 2017. DOl: https://doi. org/10.1016/j.rvsc.2017.07.010.

FISHER, M. H.; MROZIK, H. The chemistry and pharmacology of avermectins. Annual Review of Pharmacology and Toxicology, [s.I.], v. 32, p. 537-553, 1992.

GALBIATI, C. et al. Efeito de vermífugos injetáveis em bovinos de leite sobre o besouro coprófago Dichotomius anaglypticus (Mann., 1829). Ecossistema, [s.I.], v. 20, p. 100-108, 1995.

GARCIA, M. F. 35 milhões de brasileiros não têm acesso à água tratada. 2017. Disponível em: https://observatorio3setor.org.br/carrossel/35-milhoes-de-brasileiros-nao-tem-acesso-aguatratada/. Acesso em: 20 maio 2021.

HADLETT, M. et al. High concentrations of membrane-fed ivermectin are required for substantial lethal and sublethal impacts on Aedes aegypti. Parasites and Vectors, [s.I.], v. 14, 2021. DOI: https://doi. org/10.1186/s13071-020-04512-5.

IKEDA, T. Pharmacological effects of ivermectin, an antiparasitic agent for intestinal strongyloidiasis: its mode of action and clinical efficacy. Nihon Yakurigaku Zasshi, [s.l.], v. 122, n. 6, p. 527-538, 2003. DOI: https://doi.org/10.1254/fpj.122.527. 
KUESEL, A. C. Research for new drugs for elimination of onchocerciasis in Africa. International Journal for Parasitology: Drugs and Drug Resistance, [s.I.], v. 6, p. 272-286, 2016. DOI: https://doi. org/10.1016/j.ijpddr.2016.04.002.

LAING, R.; GILLAN, V.; DEVANEY, E. Ivermectin: old drug, new tricks? Trends in Parasitology, [s.I.], v. 33, n. 6, 2017. DOI: http://dx.doi.org/10.1016/j.pt.2017.02.004.

LU, J. J. et al. Multi-target drugs: the trend of drug research and development. PLoS One, [s.I.], v. 7, n. 6, p. e40262, 2012. DOl: https://doi.org/10.1371/journal.pone.0040262.

MAKHOBA, X. H. et al. Potential impact of the multi-target drug approach in the treatment of some complex diseases. Drug Design, Development and Therapy, [s.I.], v. 14, p. 3235-3249, 2020. DOI: http://doi.org/10.2147/DDDT.S257494.

MANCINI, L. et al. Need for a sustainable use of medicinal products: environmental impacts of ivermectin. Annali Istituto Superiore di Sanità, [s.I.], v. 56, p. 492-496, 2020. DOI: https://doi. org/10.4415/ANN200412.

MAS-COMA, S. et al. Sheep and cattle reservoirs in the highest human fascioliasis hyperendemic area: experimental transmission capacity, field epidemiology, and control within a one health initiative in Bolivia. Frontiers in Veterinary Science, [s.I.], v. 7, 2020, 583204. DOI: https://doi.org/10.3389/ fvets.2020.583204.

MESA, L. et al. Concentration and environmental fate of ivermectin in floodplain wetlands: An ecological approach. Science of the Total Environment, [s.I.], v. 706, 135692, 2020. DOI: https://doi. org/10.1016/j.scitotenv.2019.135692.

MOLENTO, M. B.; ARENAL, A. The breakthrough of nanotechnology to veterinary parasitology research. In: LALIOTIS, G. P. Current research in agriculture and veterinary science. London: Publisher International, 2020. chap. 6, p. 71-77. DOl: https://doi.org/10.9734/bpi/cravs/v1.

MOLENTO, M. B. Covid-19 and the rush for self-medication and self-dosing with ivermectin: a word of caution. One Health, [s.I.], v. 10, 2020. DOI: https://doi.org/10.1016/j.onehlt.2020.100148.

MOLENTO, M. B. et al. Fasciola hepatica infection in cattle and the use of simulation models for endemic areas. Journal of Helminthology, [s.I.], v. 94, e185, p. 1-9, 2020. DOI: https://doi. org/10.1017/S0022149X2000067X.

MOLENTO, M. B. et al. Partial selective treatment of Rhipicephalus microplus and breed resistance variation in beef cows in Rio Grande do Sul, Brazil. Veterinary Parasitology, [s.I.], v. 192, p. 234-239, 2013. DOI: https://doi.org/10.1016/j.vetpar.2012.10.021.

MOLENTO, M. B. Parasite control in the age of drug resistance and changing agricultural practices. Veterinary Parasitology, [s.I.], v. 163, p. 229-234, 2009. DOI: https://doi.org/10.1016/j. vetpar.2009.06.007.

OSEI-ATWENEBOANA, M. Y. et al. Phenotypic evidence of emerging ivermectin resistance in Onchocerca volvulus. Plos Neglected Tropical Diseases, [s.l.], v. 5, e998, 2011. D0I:10.1371/journal. pntd.00998. DOl: https://doi.org/10.1371/journal.pntd.0000998.

OTTENSEN, E. A.; CAMPBELL, W. C. Ivermectin in human medicine. Journal of Antimicrobials and Chemotherapy, [s.I.], v. 34, p. 195-203, 1994.

PEREGRINE, A. S. et al. Anthelmintic resistance in important parasites of horses: does it really matter? Veterinary Parasitology, [s.l.], v. 201, p. 1-8, 2014. DOl: https://doi.org/10.1016/j. vetpar.2014.01.004.

PRICHARD, R. K. Genetic variability following selection of Haemonchus contortus with anthelmintics. Trends in Parasitology, [s.I.], v. 17, p. 445-453, 2001. DOl: https://doi.org/10.1016/S1471-4922(01)01983-3. 
RAMOS, C. I. et al. Epidemiology of sheep gastrintestinal helminthosis in Planalto Catarinense region in Brazil. Ciência Rural, [s.I.], v. 34, p. 1889-1895, 2004. DOI: https://doi.org/10.1590/S010384782004000600034.

SANTOS, I. A. et al. Antivirals against coronaviruses: candidate drugs for SARS-CoV-2 treatment? Frontiers in Microbiology, [s.I.], v. 11, 1818, 2020. DOI: https://doi.org/10.3389/fmicb.2020.01818.

Sauerbrey, M.; Rakers, L. J.; Richards, F. O. Progress toward elimination of onchocerciasis in the Americas. International Health, v. 10-S1, p. 71-78, 2018. DOI: https://doi.org/10.1093/inthealth/ ihx039.

SCHAFASCHEK, A. I. I. et al. Transient threshold abundance of Haematobia irritans (Linnaeus, 1758) in cattle under integrated farming systems. International Journal of Plant Animal and Environmental Sciences, [s.I.], v. 11, p. 295-321, 2021. DOI: https://doi.org/10.26502/ijpaes.202108.

SCHMITH, V. D.; ZHOU, J.; LOHMER, L. R. L. The approved dose of ivermectin alone is not the ideal dose for the treatment of COVID-19. Clinical Pharmacology \& Therapeutics, [s.I.], v. 108, 2020. DOI: https://doi.org/10.1002/cpt.1889.

SCOPUS. Elsevier, expertly curated citation database. 2021. Disponível em: https://www.scopus. com/search/form.uri?display=basic \#basic. Acesso em: 2 mar. 2021.

SÍMSEK, S.; YAVUZ, S.; ÜNAL, S. Antiviral treatment of COVID-19, Turkish Journal of Medical Science, [s.l.], v. 50, p. 611-619, 2020. DOI: https://doi.org/10.3906/sag-2004-145.

TORGERSON, P. R. et al. World Health Organization estimates of the global and regional disease burden of 11 foodborne parasitic diseases, 2010: a data synthesis. PLoS Medicine, [s.I.], v. 12, n. 2, e1001920, 2015. DOI: https://doi.org/10.1371/journal.pmed.1001920.

TORRES-ACOSTA, J. F. J.; MOLENTO, M. B.; DE GIVES, M. Research and implementation of novel approaches for the control of nematode parasites in Latin America and the Caribbean: is there sufficient incentive for a greater extension effort? Veterinary Parasitology, [s.I.], v. 186, p. 132-142, 2012. DOI: https:// doi.org/10.1016/j.vetpar.2011.11.053.

UNITED STATES. National Institute of Health. National Library of Medicine. Disponivel em: https:// clinicaltrials.gov/ct2/results?cond=\&term=ivermectin\&cntry=\&state=\&city=\&dist=. Acesso em: 8 abr. 2021.

VERDÚ, J. R. et al. Biomagnification and body distribution of ivermectin in dung beetles. Scientific Reports, [s.l.], n. 10, 9073, 2020. DOl: https://doi.org/10.1038/s41598-020-66063-0.

WANG, D. et al. Environmental fate of the anti-parasitic ivermectin in an aquatic micro-ecological system after a single oral administration. PeerJ, [s.I.], n. 7, e7805, 2019. DOI: http://doi. org/10.7717/peerj.7805.

WEHBE, Z. et al. Repurposing ivermectin for COVID-19: molecular aspects and therapeutic possibilities. Frontiers in Immunology, [s.I.], n. 12, 663586, 2021. DOI: https://doi.org/10.3389/ fimmu.2021.663586.

ZBARSKY, V.; THOMAS, J.; GREENFIELD, S. Bioactivity of a peptide derived from acetylcholinesterase: involvement of an ivermectin-sensitive site on the alpha 7 nicotonic receptor. Neurobiology of Disease, [s.l.], v. 16, p. 283-289, 2004. DOI: https://doi.org/10.1016/j.nbd.2004.02.009. 